\title{
An investigation into the effect of depth of anesthesia on postoperative pain in laparoscopic cholecystectomy surgery: a double-blind clinical trial
}

\author{
This article was published in the following Dove Press journal: \\ Journal of Pain Research \\ 28 September 2017 \\ Number of times this article has been viewed
}

Backgrounds and objective: Some studies have shown that deeper anesthesia is more effective on postoperative analgesia and reduces the need for sedative drugs. This study sought to investigate the effect of depth of anesthesia on postoperative pain in laparoscopic cholecystectomy.

Materials and methods: In this double-blind clinical trial, 60 patients undergoing laparoscopic cholecystectomy were randomly divided into two groups: low bispectral index (L-BIS=35-44) and high bispectral index (H-BIS=45-55). Anesthesia protocol was the same for both groups (propofol and remifentanil). The pain intensity (at rest and during cough) was evaluated based on the visual analog scale scores in recovery and at 8, 16 and 24 hours after surgery.

Results: The mean pain score was significantly lower in patients in the L-BIS group at all examined times at rest and during cough than that in the H-BIS group. The number of patients in need of additional sedative drug in the H-BIS group in recovery was significantly more than that in the L-BIS group ( 27 vs 18 patients, $P=0.007$ ). The incidence of nausea in the recovery room 8 hours after the surgery was significantly less in the L-BIS group than that in the H-BIS group, while at 16 and 24 hours, no case of nausea was reported in the two groups.

Conclusion: Given the results of this study, it seems that general anesthesia with propofol and remifentanil with L-BIS causes less need for additional analgesic drug and less nausea and vomiting compared to anesthesia with H-BIS.

Keywords: BIS, postoperative pain, depth of anesthesia, laparoscopic cholecystectomy

\section{Introduction}

One outcome of surgery that all patients understand after surgery is pain. Pain is an objective feeling the severity of which depends on the individual suffering from the pain. Fear of postoperative pain is one of the most common concerns among patients and the medical team, and lack of its adequate control may lead to the release of catecholamines and metabolic hormones. They result in stress responses in the form of water and sodium retention and hemodynamic abnormalities such as sharp rise in blood pressure and tachycardia. They sometimes increase bleeding and heart attacks in elderly patients. ${ }^{1}$ Lack of pain control in postoperative period can cause delay in the onset of patients' movement, an increase in the duration of hospitalization, and interference with rehabilitation. $^{2}$ The use of appropriate methods of anesthesia and analgesia in patients undergoing surgery can be effective in reducing health costs and morbidity of patients.
Correspondence: Poupak Rahimzadeh Pain Research Center, Iran University of Medical Sciences, Hazrat Rasul Medical Complex, Niayesh Street, Sattarkan Avenue, Tehran I4456I3 I3I, Iran Email poupak_rah@hotmail.com 
Some studies have noted the effect of monitoring depth of anesthesia based on bispectral index (BIS) on postoperative pain. ${ }^{3,4} \mathrm{BIS}$ is an assay dependent on electroencephalography (EEG) introduced for monitoring depth of anesthesia and has replaced other methods such as Guedel's classification. ${ }^{5}$ This technology is sensitive to both anesthesia and consciousness. Adjusting the depth of anesthesia in adults based on BIS allows physicians to determine the amount of required anesthetic drug. Using this index can reduce the likelihood of awakening within surgery. ${ }^{6,7}$

Sahni et $\mathrm{al}^{8}$ showed that maintaining BIS $\sim 40-45$ during surgery can reduce postoperative pain and need for analgesic drug. However, the study of Baldini et al has reported conflicting results regarding BIS effect on analgesic requirement. ${ }^{9}$

Accordingly, this study sought to evaluate the effect of depth of anesthesia on the analgesia and need of patients undergoing laparoscopic cholecystectomy for painkillers.

\section{Materials and methods}

This study was performed as a double-blind clinical trial after it was approved by the ethics committee of the Iran University of Medical Sciences. The current study has been registered in the Iranian Registry of Clinical Trials (IRCT) with code $2017011412642 \mathrm{~N} 25$.

The sample size was calculated as follows: with the level of confidence of $95 \%$, ability of $80 \%$, the common standard deviation, and accuracy of 0.15 , the minimum required sample size was calculated as 24 cases in each group. We considered 30 in each group.

In all, 60 patients undergoing elective laparoscopic cholecystectomy admitted to the Hazrat Rasul Medical Hospital were enrolled in a prospective, observer-blinded, and randomized manner study. The inclusion criteria included receipt of informed consent based on participating in research project, candidate for laparoscopic cholecystectomy, age between 18 and 65 years, and patients classified as Class 1-2 in the American Society of Anesthesiologists classification. The exclusion criteria were having cancer, epilepsy, problems of hearing and the ability to communicate, BMI $>35$, heart failure, liver failure, kidney failure, sensitivity to anesthesia drugs, uncontrolled systemic disease (such as unstable diabetes), drug addiction or alcoholism, chronic pain, any kind of analgesic therapy, prolonged surgery for $>3$ hours, psychological problems, and conversion of laparoscopy to laparotomy surgery.

Patients were divided into two groups of BIS, L-BIS (35-45) and H-BIS (45-55), using block randomization. After all subjects had been assigned into blocks, randomization was performed within each block to assign subjects to one of the groups. Keeping patients in a common border of anesthesia level in both groups was hard and the anesthesia score number is changing each second, so the common border of 45 was maintained in this study for data interpretation. On arrival to the operating room, all patients were monitored by BIS, electrocardiogram (ECG), pulse oximetry, noninvasive blood pressure, and capnography. Intravenous (IV) line was embedded, and normal saline solution was administered at a rate of $5 \mathrm{~mL} / \mathrm{kg}$ as bolus for cardiovascular expansion before induction. From the induction to 10 minutes, BIS, mean arterial pressure, and end tidal $\mathrm{CO} 2$ were recorded every 2 minutes. After the first 10 minutes, these criteria were recorded every 5 minutes for 1 hour and then every 10 minutes until the outset of the surgery. During surgery, ETCO2 was maintained in the range of 30-35 mmHg. Midazolam of $0.15 \mathrm{mg} / \mathrm{kg}$ and fentanyl of $3 \mu \mathrm{g} / \mathrm{kg}$ were injected before anesthesia induction. Then, induction of general anesthesia was made with $2 \mathrm{mg} / \mathrm{kg}$ propofol and $0.2 \mathrm{mg} / \mathrm{kg}$ cisatracurium and maintained with $70-150 \mu \mathrm{g} / \mathrm{kg} / \mathrm{min}$ propofol and $0.1 \mu \mathrm{g} / \mathrm{kg} / \mathrm{min}$ remifentanil. If, during the surgery, BIS was in the desired range, while systolic blood pressure (SBP) was $>140 \mathrm{mmHg}$, trinitroglycerine infusion with the dose of 5-15 $\mu \mathrm{g} / \mathrm{min}$ was used, and if heart rate $(\mathrm{HR})>85 / \mathrm{min}$, IV labetalol up to a maximum of $30 \mathrm{mg}$ was used. If BIS was high, the dose of propofol was first increased for $10 \mu / \mathrm{kg} / \mathrm{min}$ twice, and if necessary, at the next stage, the dose of remifentanil was increased from $0.1 \mu$ / $\mathrm{kg} / \mathrm{min}$ to $0.2 \mu / \mathrm{kg} / \mathrm{min}$. Half an hour before the outset of the surgery, $1 \mathrm{~g}$ of acetaminophen and $4 \mathrm{mg}$ of ondansetron were administered. At the end of the surgery, the used propofol and duration of the surgery were recorded. The pain scores at rest and during cough were recorded at the recovery room and 8,16 , and 24 hours after the surgery using the visual analog scale (VAS) score by an investigator who was not aware of intraoperative management and BIS values of both groups, and decoding was done at the end of research.

Opioid request was recorded at the end of 24 hours. Postoperative nausea and vomiting were measured based on the scores of 0 (no nausea and vomiting), 1 (mild), 2 (moderate), and 3 (severe), and patient satisfaction was also recorded based on the scores of 0 (weak), 1 (moderate), 2 (good), 3 (very well), and 4 (excellent).

In the recovery room, patient-controlled analgesia (PCA) pump containing $500 \mu \mathrm{g}$ of fentanyl and $2 \mathrm{~g}$ of acetaminophen was set and adjusted with a flow of $4 \mathrm{~mL} /$ hour and bolus interval every 15 minutes. In patients with VAS $>3,15 \mathrm{mg}$ of meperidine was intravenously injected and recorded.

SPSS 22 software was used for data analysis, and all statistical tests were done as two tailed and with the signifi- 
cance level of 5\%. Quantitative and qualitative variables were expressed as mean $\pm \mathrm{SD}$ and number (percentage), respectively. To compare the procedure of qualitative variables at different times, repeated measures of analysis of variance (ANOVA) were used and the variable value was entered into the model as a covariate before the surgery. Other variables measured at the end of the surgery were reported along with confidence interval of $95 \%$.

\section{Results}

In this clinical trial, 60 patients undergoing elective laparoscopic cholecystectomy were assigned to the two groups of high bispectral index (H-BIS) and low bispectral index (L-BIS). Their demographic data are given in Table 1.

BIS values were compared between the two groups $(P$-value $=0.001$; Figure 1$)$. Pain intensity at rest and during cough in patients in the H-BIS group at all examined times were significantly higher than those in the L-BIS group. Figures 2 and 3 show statistically different patterns of pain

Table I Demographic variables in the examined patients in terms of the type of monitoring

\begin{tabular}{|c|c|c|c|}
\hline Variable & L-BIS & H-BIS & $P$-value \\
\hline Age (years) & $44.1( \pm 12.58)$ & $44.67( \pm 11.83)$ & 0.419 \\
\hline \multicolumn{4}{|l|}{ Gender } \\
\hline Male & 7 & 5 & 0.748 \\
\hline Female & 23 & 25 & \\
\hline Height (cm) & $162.30( \pm 7.29)$ & $162.70( \pm 8.47)$ & 0.845 \\
\hline Weight (kg) & $68.77( \pm 9.54)$ & $74.70( \pm 15.87)$ & 0.085 \\
\hline Surgical time (minutes) & $70.60( \pm \mid 3.51)$ & $73.66( \pm 10.56)$ & 0.64 \\
\hline
\end{tabular}

Note: Data presented as number or mean $( \pm S D)$.

Abbreviations: L-BIS, low bispectral index; H-BIS, high bispectral index. changes $(P$-value $=0.001)$ in patients of both groups at rest and during cough.

Total dose of used meperidine, propofol and remifentanil in both groups has been given in Table 2. The number of patients in need of meperidine in the L-BIS group was significantly lower than that in the H-BIS group $(P=0.007)$.

The mean dose of fentanyl for patients in both groups is shown in Table 2. The need for additional analgesia in the $\mathrm{H}$-BIS group was significantly higher than that in the L-BIS group ( $P=0.013$ ). There was no relationship between propofol received during the surgery and the need for additional analgesia in the first 24 hours $(P=0.348)$.

The incidence and severity of nausea during the first 24 hours after the surgery is given in Figure 4 for both groups. As shown in Figure 4, in the recovery room, the number of patients with mild and moderate nausea was higher in the $\mathrm{H}$-BIS group. In the 8th hour, all patients in the H-BIS group reported mild nausea. No nausea was reported in the H-BIS group in the 16th and 24th hour after the surgery; however, there was no nausea in the L-BIS group in none of the examined hours as well. No vomiting was recorded.

Comparing between the two groups, patients' satisfaction was higher in the L-BIS group at the time of recovery $(P=0.025)$, 8th hour $(P=0.001), 16$ th hour $(P=0.001)$, and 24 hours after the surgery $(P=0.001$; Figure 5$)$.

\section{Discussion}

Utilizing strategies pointed particularly at interrupting the mechanism responsible for pain generation such as preemptive analgesia, administration of intraoperative analgesics or both have been suggested. However, a few studies have shown

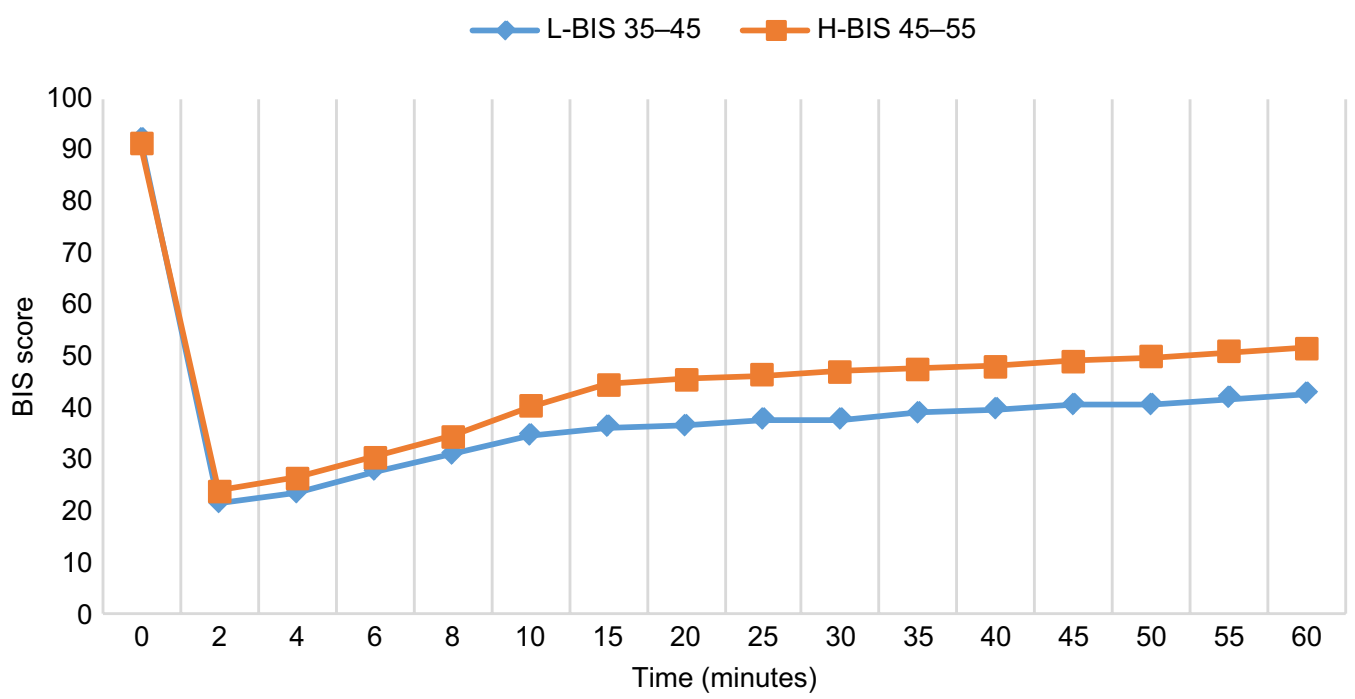

Figure I BIS graph in two groups.

Note: Keeping patients in a common border of anesthesia level in both groups was hard and the anesthesia score number is changing each second, so the common border of 45 was maintained in this study for data interpretation.

Abbreviations: BIS, bispectral index; H-BIS, high bispectral index; L-BIS, low bispectral index. 


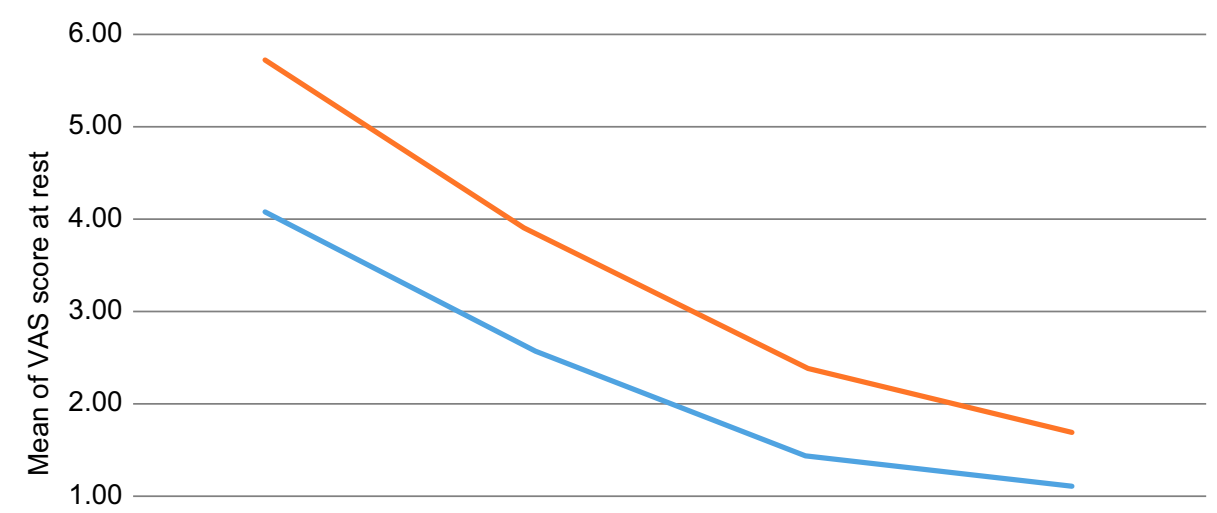

\begin{tabular}{|r|c|c|c|c|}
\cline { 2 - 5 } \multicolumn{1}{c|}{0.00} & $0 \mathrm{~h}$ & $8 \mathrm{~h}$ & $16 \mathrm{~h}$ & $24 \mathrm{~h}$ \\
\hline L L-BIS & 4.07 & 2.57 & 1.47 & 1.13 \\
\hline H-BIS & 5.70 & 3.83 & 2.40 & 1.70 \\
\hline
\end{tabular}

Figure 2 Trend diagram of mean pain intensity (VAS) at rest.

Abbreviations: VAS, visual analog scale; h, hours; L-BIS, low bispectral index; H-BIS, high bispectral index.

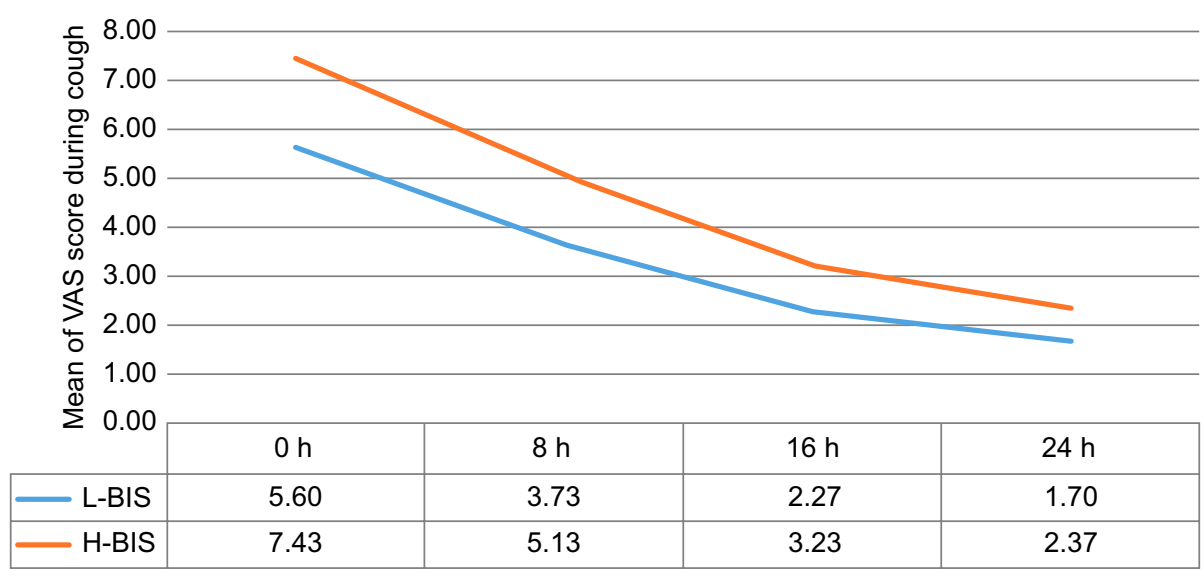

Figure 3 Trend diagram of mean pain intensity (VAS) during coughing.

Abbreviations: VAS, visual analog scale; h, hours; L-BIS, low bispectral index; H-BIS, high bispectral index.

Table 2 Type of drug injected in two groups

\begin{tabular}{llllll}
\hline Drug & Group & Patients (n) & Mean & SD & $P-\boldsymbol{V}$ \\
\hline Meperidine (mg) & L-BIS & 18 & 20.00 & 0.00 & $0.007^{*}$ \\
& H-BIS & 27 & 20.00 & 0.00 & \\
Propofol (mg) & L-BIS & 30 & 627.07 & 274.14 & 0.103 \\
& H-BIS & 30 & 624.57 & 342.69 & \\
Remifentanil (mg) & L-BIS & 30 & 986.31 & 486.55 & 0.665 \\
& H-BIS & 30 & 723.90 & 699.57 & \\
Bolus fentanyl (mg) & L-BIS & 14 & 28.57 & 17.03 & $0.013^{*}$ \\
& H-BIS & 22 & 49.09 & 25.99 & \\
\hline
\end{tabular}

Note: *Statistically significant.

Abbreviations: L-BIS, low bispectral index; H-BIS, high bispectral index.

that adequate intraoperative depth of anesthesia is associated with less postoperative pain. ${ }^{3,8}$

Based on the results, maintaining the depth of anesthesia within L-BIS could control postoperative pain better than
H-BIS from recovery time to 24 hours after the surgery and cause better analgesia for patients at rest and during cough. Maintaining L-BIS was also effective in the reduction of the incidence of nausea and vomiting after surgery for up to 8 hours. It is assumed that the greater use of propofol in the L-BIS group might influence the decreased nausea in that group. The patient's satisfaction in the L-BIS group was significantly better, which indicated the positive effects of deeper anesthesia level.

In one study, patients under laparoscopic cholecystectomy with general anesthesia in two groups with BIS $=40-45$ and BIS $=45-60$ were examined. Pain intensity and the need for analgesia were significantly lower in the first 8 hours in the lower BIS group. ${ }^{8}$ In another study on patients under anesthesia with propofol and remifentanil, patients with higher BIS values specifically requested more analgesia postoperatively. ${ }^{3}$ 


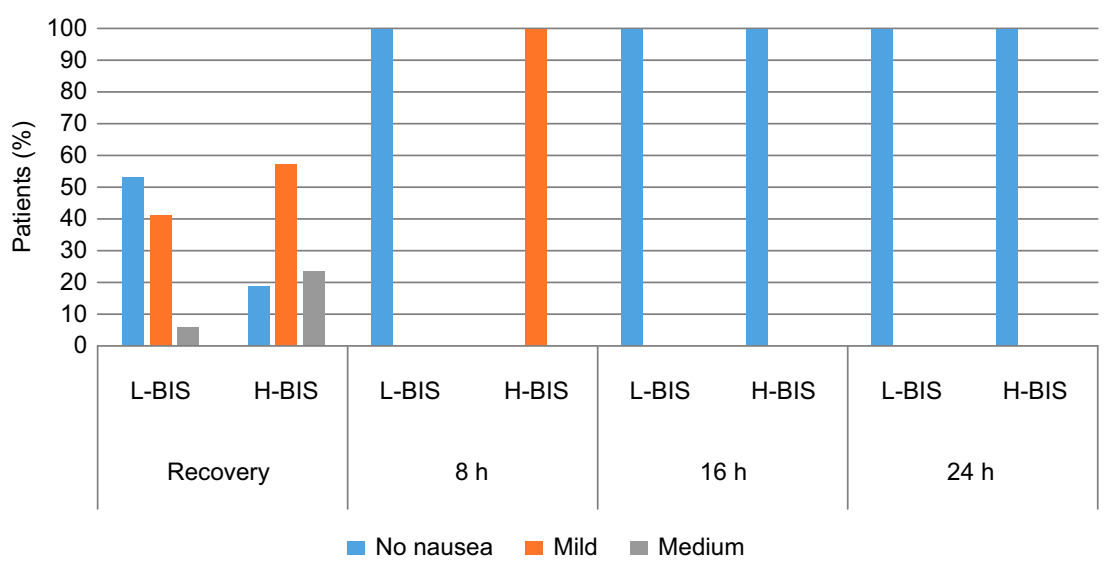

Figure 4 Bar diagram of nausea in percentage terms in the treatment groups.

Abbreviations: L-BIS, low bispectral index; H-BIS, high bispectral index; h, hours.

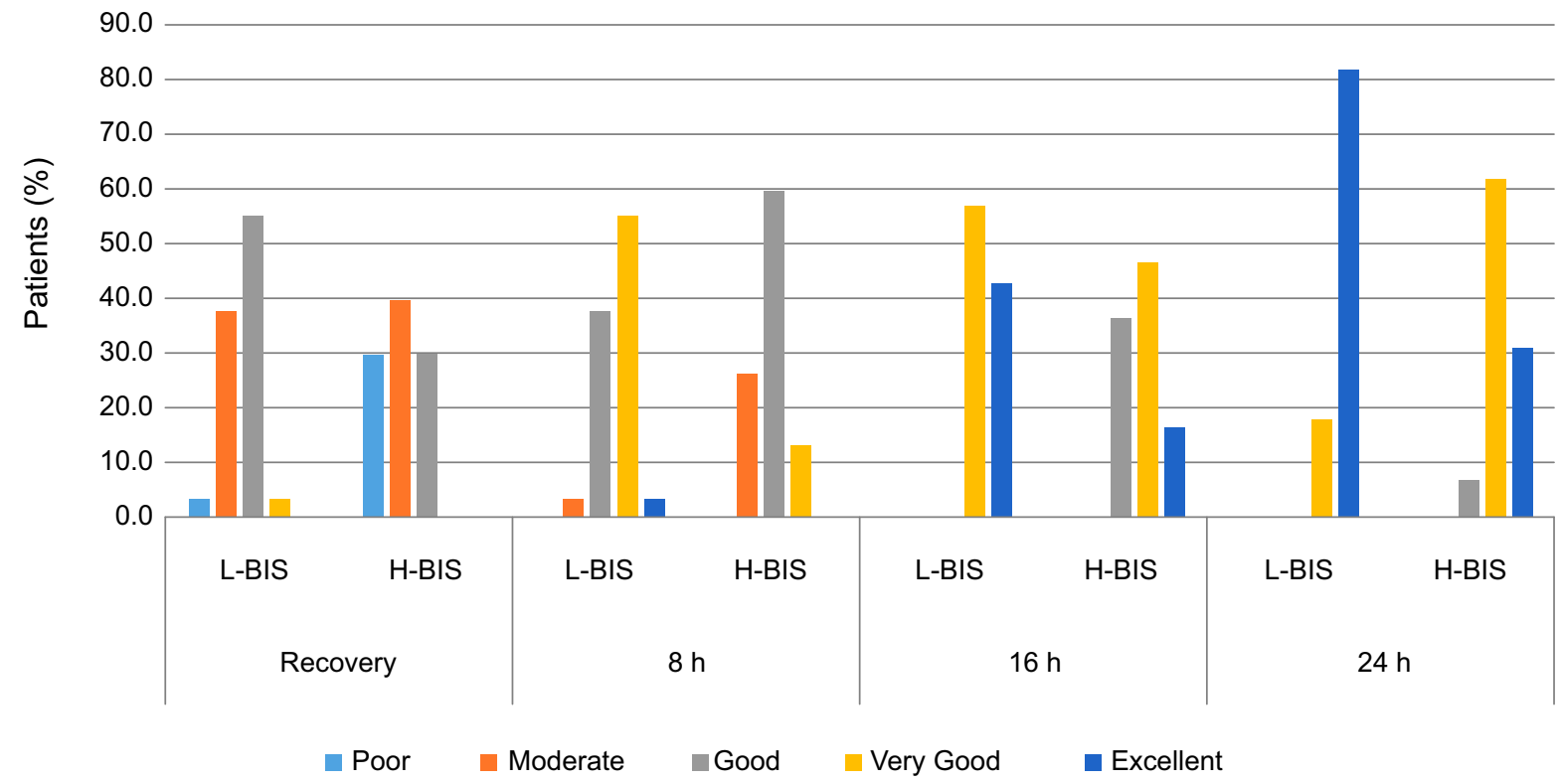

Figure 5 Satisfaction scores in both groups in terms of percentages.

Note: Modified form of Revised American Pain Society Patient Outcome Questionnaire was used.

Abbreviations: L-BIS, low bispectral index; H-BIS, high bispectral index; h, hours.

The results of the abovementioned studies were similar to the results of our study. BIS effects, especially in terms of complications, have been evaluated on long-term basis, which has shown controversial results. ${ }^{10}$

Another study chose L-BIS (20-30) and H-BIS (50-60) groups while using sevoflurane along with nitrous oxide and remifentanil for anesthesia. The researcher has concluded that a deeper anesthesia with increasing the concentration of sevoflurane leads to a decline in postoperative pain and extra analgesic need. ${ }^{11}$ Our study results showed similarity as well. Since the depth of anesthesia depends on changing the dose of drugs in each treatment protocol and not the drug type and measurement tool, ${ }^{5}$ the possibility to review and explain the possible mechanism of pain relief after surgery with increasing anesthesia depth requires further studies. In the study of Sahni et $a 1,{ }^{8}$ the impossibility to explain the pain relief mechanism in patients with deeper BIS has been studied. Another study showed that anesthesia with propofol had a better postoperative pain control and pointed out that the isoflurane-induced hyperalgesic action is due to the inhibition of nicotinic acetylcholine receptors in the brain and spinal cord, while no mechanism has been proposed for propofol. ${ }^{12}$ In this study, patients in both groups received propofol and remifentanil, and the final prescribed dose had no significant difference between them; it seems that increasing anesthesia depth has caused less pain in the L-BIS group. The study of Hand et $\mathrm{a} \mathrm{l}^{13}$ showed that the injection of subhypnotic dose of propofol could cause postoperative analgesia. In the current 
study, the analgesic request by PCA was lower in L-BIS, which cannot be caused by the effect of propofol because the final dose of propofol had no significant difference. L-BIS was achieved through administering propofol without difference in the final dose of propofol, which can be justified by longer duration of anesthesia for patients in the H-BIS group, although it was not statistically significant.

In one study, patients were evaluated with $50<\mathrm{BIS}<60$, $40<\mathrm{BIS}<50$, and $30<\mathrm{BIS}<40$ in terms of postoperative cognitive function. They concluded that $30<\mathrm{BIS}<40$ with remifentanil and propofol has little significant effect on postoperative cognitive function. ${ }^{14}$ In the study of Baldini et al, no difference was reported between the score of postoperative pain and the opioid need for patients with $\mathrm{BIS}=25$ compared to $\mathrm{BIS}=50$. No difference was observed in the levels of cortisol, glucose, and lactate in both groups. ${ }^{9}$ Based on the results of Law et al, increasing the anesthesia depth $(\mathrm{BIS}<25)$ is not a clinically useful strategy for reducing postoperative pain. The differences between the study by Law et $\mathrm{al}^{15}$ and the previous studies were related to larger sample size, considering different types of surgeries with different pain scores and lack of using nitrous oxide as an analgesic during anesthesia. In the study by Sahni et al, the group with lower BIS had less nausea, which was in line with our results. It is presumed that less nausea is associated with less usage of narcotic drugs in the L-BIS group. ${ }^{16}$ Using BIS on hemodynamics and recovery profile, especially in pediatric patients, has been recommended as well. The associations between anesthesia depth and death and myocardial infarction or postoperative cognitive decline and long-term survival have been studied, but the available evidence is inconclusive. ${ }^{17,18}$

\section{Conclusion}

Given the results of this study, it seems that general anesthesia with propofol and remifentanil with L-BIS causes less need for additional analgesia and less nausea and vomiting compared to anesthesia with H-BIS.

\section{Author contributions}

Poupak Rahimzadeh contributed to study concept and design, Seyed Hamid Reza Faiz to acquisition of data, Seyed Alireza Seyed Siamdoust to analysis and interpretation of data, Seyed Hamid Reza Faiz, Leila Houshmand, and Poupak Rahimzadeh to overview of the manuscript for important intellectual content, Poupak Rahimzadeh and Seyed Alireza Seyed Siamdust to administrative, technical, and material support, and Poupak Rahimzadeh to study supervision. All authors contributed toward data analysis, drafting and criti- cally revising the paper, gave final approval of the version to be published, and agree to be accountable for all aspects of the work.

\section{Disclosure}

The authors report no conflicts of interest in this work.

\section{References}

1. Sedlář M, Kvasnička J, Krška Z, Tománková T, Linhart A. Early and subacute inflammatory response and long-term survival after hip trauma and surgery. Arch Gerontol Geriatr. 2015;60(3):431-436.

2. Kehlet H. The endocrine-metabolic response to postoperative pain. Acta Anaesthesiol Scand Suppl. 1982;74:173-175.

3. Henneberg SW, Rosenberg D, Jensen EW, Ahn P, Burgdorff B, Thomsen LL. Peroperative depth of anaesthesia may influence postoperative opioid requirements. Acta Anaesthesiol Scand. 2005;49(3):293-296.

4. Gurman GM, Popescu M, Weksler N, Steiner O, Avinoah E, Porath A. Influence of the cortical electrical activity level during general anaesthesia on the severity of immediate postoperative pain in the morbidly obese. Acta Anaesthesiol Scand. 2003;47(7):804-808.

5. Sigl JC, Chamoun NG. An introduction to bispectral analysis for the electroencephalogram. J Clin Monit. 1994;10(6):392-404.

6. Johansen JW, Seber PS. Development and clinical application of electroencephalographic bispectrum monitoring. Anesthesiology. 2000;93(5):1336-1344.

7. Punjasawadwong Y, Phongchiewboon A, Bunchungmongkol N. Bispectral index for improving anaesthetic delivery and postoperative recovery. Cochrane Database Syst Rev. 2007;4:CD003843.

8. Sahni N, Anand LK, Gombar K, Gombar S. Effect of intraoperative depth of anesthesia on postoperative pain and analgesic requirement: a randomized prospective observer blinded study. J Anaesthesiol Clin Pharmacol. 2011;27(4):500-505.

9. Baldini G, Bagry H, Carli F. Depth of anesthesia with desflurane does not influence the endocrine-metabolic response to pelvic surgery. Acta Anaesthesiol Scand. 2008;52(1):99-105.

10. Short TG, Leslie K, Campbell D, et al. A pilot study for a prospective, randomized, double-blind trial of the influence of anesthetic depth on long-term outcome. Anesth Analg. 2014;118(5):981-986.

11. Fritz BA, Rao P, Mashour GA, et al. Postoperative recovery with bispectral index versus anesthetic concentration-guided protocols. Anesthesiology. 2013;118(5):1113-1122.

12. Cheng SS, Yeh J, Flood P. Anesthesia matters: patients anesthetized with propofol have less postoperative pain than those anesthetized with isoflurane. Anesth Analg. 2008;106(1):264-269.

13. Hand R, Riley GP, Nick ML, Shott S, Faut-Callahan M. The analgesic effects of subhypnotic doses of propofol in human volunteers with experimentally induced tourniquet pain. AANA J. 2001;69(6): 466-470.

14. Zhang D, Nie A. Assessment of different anesthesia depth under total intravenous anesthesia on postoperative cognitive function in laparoscopic patients. J Res Med Sci. 2016;21:73-77.

15. Law CJ, Jacobson GM, Kluger M, Chaddock M, Scott M, Sleigh JW. Randomized controlled trial of the effect of depth of anaesthesia on postoperative pain. Br J Anaesth. 2014;112(4):675-680.

16. Rorarius MG, Mennander S, Suominen P, et al. Gabapentin for the prevention of postoperative pain after vaginal hysterectomy. Pain. 2004;110(1-2):175-181.

17. Sargin M, Uluer MS, Ozmen S. The effects of bispectral index monitoring on hemodynamics and recovery profile in developmentally delayed pediatric patients undergoing dental surgery. Paediatr Anaesth. 2015;25(9): 950-955.

18. Leslie K, Short TG. Anesthetic depth and long-term survival: an update. Can J Anaesth. 2016;63(2):233-240. 
The Journal of Pain Research is an international, peer reviewed, open access, online journal that welcomes laboratory and clinical findings in the fields of pain research and the prevention and management of pain. Original research, reviews, symposium reports, hypothesis formation and commentaries are all considered for publication
The manuscript management system is completely online and includes a very quick and fair peer-review system, which is all easy to use. Visit http://www.dovepress.com/testimonials.php to read real quotes from published authors. 